\title{
Sorção e solubilidade de cimentos endodônticos biocerâmicos e cimento à base de resina epóxica
}

\author{
Sorption and solubility of bioceramic endodontic sealers and sealer based on epoxy resin
}

Sorción y solubilidad del cemento endodóntico biocerámico y del cemento a base de resina epóxica

Recebido: 31/05/2021 | Revisado: 06/06/2021 | Aceito: 08/06/2021 | Publicado: 23/06/2021

\author{
Lucas Adriano Pascuti Bastos \\ ORCID: https://orcid.org/0000-0001-5365-7442 \\ Fundação Herminio Ometto, Brasil \\ E-mail: lucas-apb@hotmail.com \\ Rafael Pino Vitti \\ ORCID: https://orcid.org/0000-0001-6366-5868 \\ Fundação Herminio Ometto, Brasil \\ E-mail: rafaelvitti@ fho.edu.br \\ Homero Casonato Junior \\ ORCID: https://orcid.org/0000-0002-1341-4363 \\ Fundação Herminio Ometto, Brasil \\ E-mail: casonatojr@gmail.com
}

\begin{abstract}
Resumo
Introdução: Os cimentos endodônticos são utilizados na obturação do canal radicular e estão associados ao sucesso do tratamento endodôntico, preenchendo os espaços entre as paredes do canal radicular e a guta-percha. A sorção e a solubilidade desses materiais são propriedades que estão relacionadas ao processo de remineralização dos tecidos periapicais e à capacidade antimicrobiana, além de serem parâmetros ligados a efeitos deletérios, tal como a degradação do cimento e a consequente recontaminação do canal radicular. Métodos: os cimentos AH Plus (Dentsply, São Paulo, SP, Brasil), MTA Fillapex (Angelus, Londrina, PR, Brasil) e Bio-C Sealer (Angelus, Londrina, PR, Brasil) foram manipulados seguindo as instruções do fabricante e armazenados em estufa a $37^{\circ}$. Posteriormente, foram pesados em balança analítica de precisão para que então as amostras fossem colocadas em água destilada por 24 horas em estufa a $37^{\circ}$ e dessa forma, após remoção do excesso as amostras passaram pelos testes de sorção e solubilidade, sendo as médias comparadas pelo teste de Tukey HSD. Resultados: relativo à sorção foi observado que o MTA Fillapex apresentou o maior valor, já os outros cimentos tiveram menores valores e sem diferenças estatisticamente significantes entre si. O cimento AH-Plus apresentou o menor valor de solubilidade, enquanto o cimento Bio- $\mathrm{C}$ o maior. Conclusões: Mesmo com valores maiores que o preconizado pelas normas ISO, os cimentos Bio-C e MTAFillapex ainda assim são adequados para uso clínico.
\end{abstract}

Palavras-chave: Endodontia; Solubilidade; Obturação do canal radicular.

\begin{abstract}
Introduction: Endodontic cements are used in root canal filling and are associated with successful endodontic treatment, filling the spaces between the walls of the root canal and gutta-percha. The sorption and solubility of these materials are properties that are related to the process of remineralization of periapical tissues and antimicrobial capacity, in addition to being parameters linked to deleterious effects, such as cement degradation and the consequent recontamination of the root canal. Methods: AH Plus cements (Dentsply, São Paulo, SP, Brazil), MTA Fillapex (Angelus, Londrina, PR, Brazil) and Bio-C Sealer (Angelus, Londrina, PR, Brazil) were handled following the manufacturer's instructions and stored in an oven at $37^{\circ}$. Subsequently, they were weighed on a precision analytical balance so that the samples were then placed in distilled water for 24 hours in an oven at $37^{\circ}$ and thus, after removing the excess, the samples underwent sorption and solubility tests, and the averages were compared by Tukey HSD test. Results: regarding sorption, it was observed that MTA Fillapex had the highest value, whereas the other cements had lower values and no statistically significant differences between them. AH-Plus cement had the lowest solubility value, while Bio- $\mathrm{C}$ cement had the highest. Conclusions: Even with values higher than those recommended by ISO standards, Bio-C and MTA-Fillapex cements are still suitable for clinical use.
\end{abstract}

Keywords: Endodontics; Solubility; Root canal filling.

\section{Resumen}

Introducción: Los cementos endodónticos se utilizan en el relleno del conducto radicular y se asocian con un tratamiento endodóntico exitoso, llenando los espacios entre las paredes del conducto radicular y la gutapercha. La sorción y solubilidad de estos materiales son propiedades que se relacionan con el proceso de remineralización de los 
tejidos periapicales y la capacidad antimicrobiana, además de ser parámetros ligados a efectos deletéreos, como la degradación del cemento y la consecuente recontaminación del conducto radicular. Métodos: Los cementos AH Plus (Dentsply, São Paulo, SP, Brasil), MTA Fillapex (Angelus, Londrina, PR, Brasil) y Bio-C Sealer (Angelus, Londrina, PR, Brasil) se manipularon siguiendo las instrucciones del fabricante y se almacenaron en un horno a $37{ }^{\circ}$. Posteriormente, se pesaron en una balanza analítica de precisión para luego colocar las muestras en agua destilada durante 24 horas en un horno a $37^{\circ}$ y así, luego de retirar el exceso, las muestras se sometieron a pruebas de sorción y solubilidad, y se compararon los promedios. por la prueba Tukey HSD. Resultados: con respecto a la sorción, se observó que MTA Fillapex tuvo el valor más alto, mientras que los otros cementos tuvieron valores más bajos y sin diferencias estadísticamente significativas entre ellos. El cemento AH-Plus tuvo el valor de solubilidad más bajo, mientras que el cemento Bio-C tuvo el más alto. Conclusiones: Incluso con valores superiores a los recomendados por las normas ISO, los cementos Bio-C y MTA-Fillapex siguen siendo adecuados para uso clínico.

Palabras clave: Endodoncia; Solubilidad; Obturación del conducto radicular.

\section{Introdução}

Os cimentos endodônticos são utilizados na obturação do canal radicular e mantêm relação direta com o sucesso do tratamento endodôntico, uma vez que apresentam em sua composição materiais e compostos químicos que influenciarão suas propriedades de biocompatibilidade, citotoxidade, infiltração, manchamento, sorção e solubilidade. (Dourado, 2017). Dessa forma, estabelecendo o preenchimento de espaços entre as paredes do canal e o cone de guta-percha, promovendo um vedamento adequado impedindo a contaminação do canal por agentes intrínsecos ou extrínsecos que possam penetrar pelos espaços em que o material obturador semissólido não alcança (Zvi, et al., 2011; Zhou, et al., 2013).

Os cimentos endodônticos biocerâmicos são materiais hidrofílicos que apresentam propriedades muito favoráveis para sucesso do tratamento, selamento de perfurações e reabsorções radiculares, como baixa toxicidade, elevado $\mathrm{pH}$, capacidade de formação de hidroxiapatita, capacidade de induzir resposta regenerativa, biocompatibilidade e bioatividade (Lima, et al., 2017; Dourado, 2017). Já com relação aos materiais à base de resina epóxi, de acordo com Zvi et al., 2011, possuem uma boa viscosidade e tempo de trabalho adequado, além disso a formulação do AH-Plus não há liberação de formaldeído após a presa e possui boa estabilidade dimensional, contudo, Zordan-Bronzel et al., 2019, relata que o material não apresenta propriedades biotativas.

Sorção é uma propriedade que avalia a perda de substância de um material durante sua fase de presa desde a fase líquida até a fase sólida. Essa propriedade abrange as propriedades físicas de adsorção, absorção e precipitação, ocorrendo simultaneamente (Nascimento Sobrinho, 2014), diferente da solubilidade que é a capacidade de uma substância se dissolver quando em contato com outra e para que a interação ocorra são necessários dois elementos nesse processo, o soluto e o solvente (Martins, et al., 2013).

Essas propriedades físicas exercem papel importante no processo de cura ou não do elemento tratado, pois a solubilidade juntamente com a sorção podem promover um ambiente favorável ao reparo com potencial de remineralização e antimicrobiano por meio da liberação de íons, entretanto, os materiais que possuem alta solubilidade tendem a aumentar a probabilidade de desintegração, portanto podem gerar um ambiente desfavorável ao reparo, além de possibilitar a degradação do cimento e a consequente contaminação do espaço do canal radicular (Razdan, et al., 2019; Zhou, et al., 2013).

Diante do exposto, pode-se afirmar que a sorção e a solubilidade são propriedades importantes de serem conhecidas e estudadas pelo profissional para buscar pelo material que seja o mais ideal possível, visto que, se houver a degradação do cimento endodôntico, ele deixará de manter o vedamento e, por conseguinte, haverá grande possibilidade de contaminação do conduto e falha do tratamento endodôntico.

O objetivo do estudo foi avaliar as capacidades de sorção e solubilidade do cimento endodôntico biocerâmico Bio-C (Angelus, PR, Brasil) em comparação a dois diferentes cimentos endodônticos, o primeiro à base de resina epóxi (AH-Plus), outro à de mineral trióxido agregado (MTA-Fillapex). 


\section{Metodologia}

Para a metodologia, foi utilizada a ISO 6876, a qual descreve que os materiais que não necessitam de água para a presa devem ser colocados sobre placa de vidro e sobre o material deve ser colocada outra placa de vidro com uma folha plástica, para que, dessa forma as amostras sejam planificadas e colocados em estufa por tempo 50\% maior do que o indicado pelo fabricante. Já para os materiais que necessitam de umidade será preciso a mistura de $2 \mathrm{~g}$ de material junto com $0,02 \mathrm{ml}$ de água destilada e as amostras devem ser planificadas da mesma forma como para materiais que não precisam de umidade e então devem ser colocados em estufa por 24 horas. Após a presa, os materiais devem ser colocados em recipiente com água destilada e colocados em estufa por 24 horas. A diferença entre a massa original e a massa final deve ser registrada, com a aproximação de $0,001 \mathrm{~g}$.

Contudo, alguns pontos da ISO foram alterados para a pesquisa em questão visto que a adição de $0,02 \mathrm{ml}$ de água destilada ao material fez com que o cimento não tomasse presa, dessa forma a umidade relativa foi criada por meio de recipientes plásticos com gazes úmidas.

A manipulação dos materiais (Tabela 1) foi feita de acordo com as especificações do fabricante. Dessa forma, os cimentos AH-Plus e MTA-Fillapex foram manipulados sobre placa de vidro utilizando espátula metálica para fazer a mistura de ambas as pastas por 30 segundos, as quais foram proporcionadas igualmente (1:1), até uma consistência homogênea ser obtida, já o cimento Bio-C foi proporcionado com o auxílio de pontas aplicadoras, as quais apresentam uma espiral que faz a mistura do material, pois internamente a seringa é composta por dois tubos com as pastas, assim a pressão exercida pelo êmbolo faz com que os materiais passem pelas espirais da ponta aplicadora, dessa forma fazendo a mistura, e obtendo um resultado homogêneo.

Em seguida, os cimentos foram inseridos com leve excesso em matrizes de silicone por condensação com 3,7 mm de diâmetro interno e 1,9 $\mathrm{mm}$ de espessura. Sobre o conjunto matriz-cimento foi pressionado a ponta ativa da espátula metálica para estabilização planificação da superfície da amostra. Para o cimento AH-Plus, o conjunto matriz-cimento foi colocado sobre tira de poliéster apoiados em placa de vidro e permaneceram por 72 horas em estufa a $37^{\circ} \mathrm{C}$. Já os cimentos MTAFillapex e Bio-C foram dispostos nas matrizes de silicone sobre tira de poliéster e apoiados em parte do bloco de espatulação, então por não haver estufa com umidade relativa na instituição foi necessário criar essa umidade de maneira alternativa, dessa forma foram colocadas 7 gazes úmidas dentro do recipiente plástico, colocou-se o conjunto matriz-cimento sobre as gazes e então foram colocadas mais 2 gazes úmidas sobre o material, o recipiente foi fechado e permaneceu em estufa 72 horas a $37^{\circ} \mathrm{C}$ e assim foi possível manter a umidade relativa.

Após a presa dos cimentos, foram cuidadosamente removidos da matriz seccionando-a com lâmina de bisturi e foram pesados em balança analítica com precisão de 0,001g, até obter uma massa constante, assim definindo o peso inicial. Em seguida, as amostras foram colocadas dentro de recipientes plásticos com $30 \mathrm{ml}$ de água destilada para os testes de sorção e solubilidade $(n=6)$, e permaneceram por 24 horas em estufa a $37^{\circ} \mathrm{C}$. Para o completo contato das superfícies dos cimentos com a água destilada, as amostras foram suspensas por suporte de borracha dentro do recipiente plástico, evitando o contato entre as superfícies das amostras e do recipiente onde estavam armazenadas com a água destilada.

Após 24 horas as amostras foram removidas, colocadas sobre papel absorvente para remoção do excesso de água e pesadas para obtenção do peso úmido para as amostras do teste de sorção. Em seguida, as amostras foram secas utilizando estufa a $110^{\circ} \mathrm{C}$ por 2 horas e colocadas em dessecador por 30 minutos para realizar uma nova pesagem para solubilidade (peso seco).

Todas as pesagens foram realizadas 3 vezes até a obtenção de uma massa constante $(0,001 \mathrm{~g})$ e as amostras que demonstraram evidências de defeito ou desintegração foram descartadas e o teste refeito.

O resultado foi obtido por meio de cálculos para sorção e solubilidade e para a análise estatística, os dados foram 
analisados pelo teste paramétrico de Kolmogorov-Smirnov e por ANOVA-1 fator, sendo as médias comparadas pelo teste de Tukey HSD $(\alpha=0,05)$.

A sorção aparente de água, foi calculada por meio da equação: Sorção $=\left[\frac{\text { peso umido-peso inicial }}{\text { peso inierial }}\right] x 100 \%$
A solubilidade das amostras, foi calculada utilizando a equação: Solubilidade $=\left[\frac{\text { peso seco-peso inicial }}{\text { peso inicial }}\right] x 100 \%$

Tabela 1. Cimentos endodônticos.

\begin{tabular}{|c|c|c|c|}
\hline Nome Comercial & Fabricante & Composição Química & Lote \\
\hline AH-Plus & Dentsply, RJ, Brasil & $\begin{array}{l}\text { Pasta A: resina epóxi de bisfenol-a; resina epóxi de } \\
\text { bisfenol-f; tungstato de cálcio; óxido de zircônio; sílica } \\
\text { e óxido de ferro } \\
\text { Pasta B: amina adamantada; n, n”-dibenzil-5- } \\
\text { oxanonane diamina-1,9; tcd-diamina; tungstato de } \\
\text { cálcio; óxido de zircônio; sílica e óleo de silicone. }\end{array}$ & $364801 \mathrm{~L}$ \\
\hline Bio-C & Angelus, PR, Brasil & $\begin{array}{l}\text { Silicatos de cálcio, aluminato de cálcio, óxido de } \\
\text { cálcio, óxido de zircônio, óxido de ferro, dióxido de } \\
\text { silício e agente de dispersão. }\end{array}$ & 101582 \\
\hline MTA-Fillapex & Angelus, PR, Brasil & $\begin{array}{l}\text { Pasta à base: resina salicilato, resina natural, tungstato } \\
\text { de cálcio, sílica nano particulada, pigmentos; } \\
\text { Pasta Catalisadora: Resina diluente, mineral trióxido } \\
\text { agregado, sílica nano particulada, pigmentos. }\end{array}$ & 101439 \\
\hline
\end{tabular}

Fonte: Bula dos materiais.

\section{Resultados}

De acordo com os testes laboratoriais realizados, é possível observar que o cimento à base de resina epóxica (AHPlus) obteve os menores valores de solubilidade, diferente dos cimentos MTA-Fillapex e Bio-C que demonstraram sofrerem mais com essa propriedade, no entanto, referente a sorção, o cimento Bio-C apresentou o menor resultado entre os materiais testados (Tabela 2 e Figura 1).

Tabela 2. Médias $( \pm \mathrm{DP})$ da sorção e solubilidade dos cimentos estudados.

\begin{tabular}{lll}
\hline & Sorção & Solubilidade \\
\hline AH-Plus & $3,95(2,07) \mathrm{B}$ & $1,22(1,12) \mathrm{C}$ \\
MTA-Fillapex & $13,22(4,27) \mathrm{A}$ & $24,87(4,13) \mathrm{B}$ \\
Bio-C Sealer & $3,61(1,57) \mathrm{B}$ & $37,39(3,35) \mathrm{A}$ \\
\hline
\end{tabular}

Nota: Letras diferentes indicam diferenças estatisticamente significantes dentro de cada teste (coluna) $(\rho<0,05)$. Fonte: Autores. 
Figura 1. Resultado gráfico dos valores de Sorção e Solubilidade.

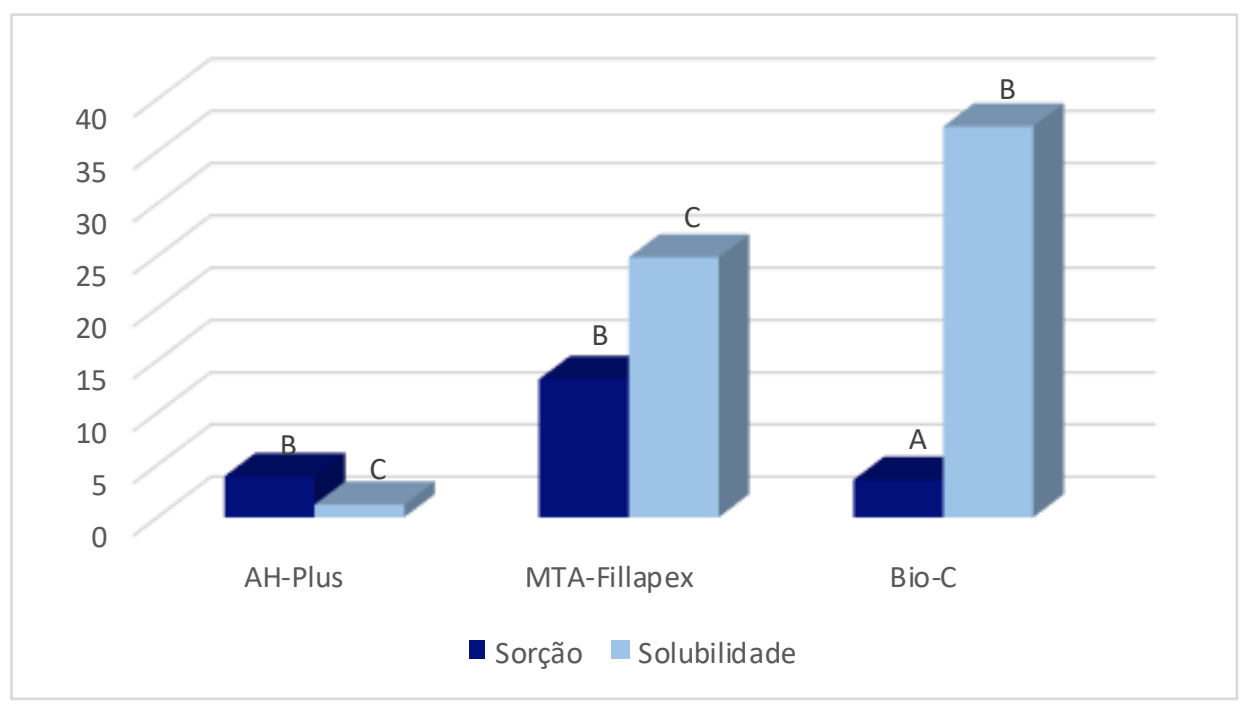

Fonte: Autores.

\section{Discussão}

Os cimentos endodônticos são importantes no procedimento de obturação do canal radicular, pois, em consonância a guta-percha, mantêm o vedamento tridimensional do espaço do sistema de canais radiculares e promovem um melhor prognóstico. São essenciais ao tratamento, afinal o material permeia por ramificações e irregularidades dos canais onde o material obturador sólido não alcança (De Deus, 2002).

O material obturador biocerâmico MTA-Fillapex, segundo o fabricante, tem por base o mineral trióxido agregado, que confere características ideais para o tratamento endodôntico e de acordo com Sousa, et al., (2014), é um bom material para aplicações clínicas, apresentando bons resultados em tecidos periapicais, bem como capacidade de indução a biomineralização, uma vez que possui alta liberação de cálcio. Contudo, Oliveira (2017) relata que embora o material possua boa capacidade mineralizadora, o bismuto presente em sua composição pode provocar manchas dentais, por conta de sua interação com o colágeno e com o hipoclorito utilizado no tratamento endodôntico.

Já o cimento AH-Plus, de acordo com o fabricante, tem como base a resina epóxi-amina, o que confere boas propriedades ao material, como alta radiopacidade, propriedades autoadesivas, boa estabilidade dimensional e propriedade de vedação a longo prazo. Zordan-Bronzel, et al., (2019), relata que por conta das propriedades químicas e físicas do cimento AHPlus ele é considerado padrão ouro e possui baixa solubilidade, que está relacionada com as fortes ligações cruzadas da resina epóxi, porém o material não possui propriedades bioativas. De acordo com Alonso (2005), o cimento AH-Plus possui um adequado poder de escoamento, em consonância a isso Scelza (2006) cita que o escoamento de um cimento endodôntico é de grande importância, visto que quanto maior for seu poder de escoamento, maior será seu potencial de preenchimento

O cimento endodôntico Bio-C possui polietilenoglicol em sua formulação, o que proporciona boa biocompatibilidade, além de uma fácil limpeza uma vez que este material é hidrossolúvel. Santos (2019) cita que o silicato de cálcio presente em sua composição promove um bom comportamento bioativo, assim como também possui boas propriedades químicas, físicas e biológicas, o que proporciona a capacidade de induzir reação osteogênica.

Segundo a norma ISO 6876, a solubilidade dos cimentos endodônticos não devem ultrapassar 3\% após 24 horas, assim, de acordo com os resultados o cimento AH-Plus demonstrou o menor valor de solubilidade, sendo ele em média 1,22\%. Torres (2016), sugere que as ligações cruzadas que ocorrem entre os polímeros resinoso do cimento AH-Plus promovem sua baixa solubilidade, assim como Viapiana, et al., (2013), relaciona que a resistência a compressão tem influência nessa 
propriedade física do material, portanto o baixo valor de solubilidade do cimento AH-Plus pode ser em decorrência de sua alta resistência a compressão que ocorre pelas suas fortes ligações entre os monômeros da resina em sua composição. Os demais materiais apresentaram solubilidade maior do que 3\%, sendo que o MTA-Fillapex $(24,87 \%)$ e Bio-C $(37,39 \%)$, demonstraram os maiores valores.

Em um estudo feito por Viapiana, et al., (2013), foi mostrado que componentes da própria composição do MTAFillapex podem diminuir sua resistência a compressão e essa baixa resistência pode estar relacionada a uma maior solubilidade em decorrência da diminuição do carbono e essa diminuição do carbono se dá por conta da degradação do polímero, onde ocorre a perda da matriz e por consequência resulta na diminuição de resistência mecânica.

De acordo com Coomaraswamy, et al., (2007), a resistência a compressão é um fator que indica a durabilidade e, portanto, a longevidade de um cimento e de acordo com um estudo feito por Viapiana et al., (2013), foi relatado que a resistência a compressão pode ser afetada por diversos fatores, dentre eles estão o tamanho das partículas radiopacificadoras, fortes ligações entre os monômeros da resina do cimento AH-Plus, assim como uma alta solubilidade do cimento MTAFillapex pode diminuir essa resistência.

A alta solubilidade do cimento Bio-C pode estar relacionada as nano partículas hidrofílicas que estão presentes neste material, o que aumenta a área superficial, assim permitindo que uma maior quantidade de moléculas liquidas entrem em contato com o cimento obturador (Mendes, 2018).

O cimento MTA-Fillapex apresentou o maior valor no teste de sorção, enquanto os demais materiais tiveram valores menores e sem diferenças estatisticamente significantes entre si, porém mesmo que o valor de sorção tenha se mostrado elevado, Dourado (2017), sugere que o cimento MTA-Fillapex possui uma boa liberação de cálcio e isso favorece a indução a biomineralização, sendo assim, é adequado para o uso clínico. E de acordo com Vitti, et al., (2013) e Torres (2016), o óxido de bismuto tem sido relacionado a uma diminuição da estabilidade molecular em materiais feitos à base de MTA, pelo fato de que pode provocar porosidades na matriz da resina após ocorrer a solubilidade, com isso facilita a absorção de água, dessa forma esse fator pode estar ligado a alta sorção do cimento MTA-Fillapex. Dourado (2017), demonstrou que o cimento MTAFillapex possui alta liberação de cálcio, o que é de grande relevância clínica, tendo em vista que por meio dessa liberação é possível que ocorra a indução da biomineralização

Segundo a norma ISO 6876, o cimento Bio-C ultrapassou o limite de 3\% de solubilidade, o que também foi relatado no estudo feito por Zordan-Bronzel, et al., (2019), onde a solubilidade desse material foi maior do que $10 \%$. Assim o autor sugere que a solubilidade dos materiais à base de silicato de cálcio se dá por conta da liberação de íons $\mathrm{OH}-\mathrm{Ca}^{2}+\mathrm{e}$ a explicação do alto valor obtido está relacionada ao aumento da área superficial, que ocorre por conta de suas nanopartículas hidrofílicas, dessa forma permite um maior contato das moléculas liquidas com o material obturador. No entanto, por mais que a solubilidade seja alta, os cimentos produzidos à base de silicato de cálcio têm um bom potencial bioativo como resultado da solubilidade desses materiais.

Com relação a sorção, o cimento Bio-C apresentou a menor porcentagem dentre os materiais avaliados, já os testes de solubilidade demonstraram que apenas o cimento AH-Plus apresentou valores de solubilidade aceitável de acordo com as normas da ISO 6876, diferente do MTA-Fillapex e Bio-C que apresentaram altos valores dessa propriedade, sendo o Bio-C o mais afetado pela solubilidade. No entanto, mesmo com o alto valor de solubilidade do cimento Bio-C, esse material é adequado para o uso clínico, assim como o cimento MTA-Fillapex que apresentou altos valores de sorção. Isso se dá pelo fato de que o estudo realizado, seguindo as normas da ISO 6876, são feitos em contato direto com a água, o que difere da umidade que a dentina possui, podendo assim resultar diferentes valores um estudo in vitro da prática clínica in vivo. Assim, ainda que houveram valores acima do preconizado pela ISO, todos os materiais são adequados para a utilização clínica. 


\section{Conclusão}

De acordo com os resultados obtidos, foi possível verificar que o cimento Bio-C apresentou resultados aceitáveis de sorção, contudo um alto valor de solubilidade, acima do preconizado pela ISO 6876, já o cimento MTA-Fillapex apresentou o maior valor de sorção, porém clinicamente aceitável e o cimento obturador à base de resina epóxica, AH-Plus, foi o menos afetado pela sorção e solubilidade, estando seus resultados dentro da ISO 6876.

\section{Referências}

Alonso, F. S., Gomes, C. C., Freitas, L. F., Gomes, I. C., Pinto, S. S. \& Penina, P. (2005). Análise comparativa do escoamento de dois cimentos endodônticos: Endofil e AH-Plus. UFES revista de odontologia, 7(1), 48-54, 2005. https://docs.bvsalud.org/biblioref/2019/02/689141/analise.pdf

Coomaraswamy, K. Lumley, P. \& Hofmann, M. (2007) Effect of Bismuth Oxide Radioopacifier Content on the Material Properties of an Endodontic Portland Cement-based (MTA-like) System. Journal of Endodontics, 33(3), 295-298. 10.1016/j.joen.2006.11.018

De Deus, G., Gurgel Filho, E. D., Ferreira, C. M. \& Coutinho Filho, T. (2002). Penetração intratubular de cimentos endodônticos. Pesquisa Odontológica Brasileira, 16(4), 332-336. 10.1590/S1517-74912002000400009

Dourado TTH (2017). Análise da literatura científica especializada das propriedades físicas, químicas e biológicas dos cimentos biocerâmicos. [Trabalho de conclusão de curso, Universidade Federal da Paraíba]. https://repositorio.ufpb.br/jspui/handle/123456789/12478

Grech, L., Mallia, B. \& Camilleri, J. (2013). Investigation of the physical properties of tricalcium silicate cement-based root-end filling materials. Dent Mater, 29(2), 20-28. 10.1016/j.dental.2012.11.007

International Organization for Standardization. (2012). Dental Root Canal Sealing Materials (ISO Standard No. 6876:2012).

Kopper, P. M. P. (2008). Estudo da capacidade de selamento do AH-Plus, Endorez e Real Seal, em canais radiculares de cães expostos ao meio bucal por meio da avaliação da resposta inflamatória periapical. [Doutorado em Clínica Odontológica - Área de Concentração Endodontia, Universidade Federal do Rio Grande do Sul]. https://www.lume.ufrgs.br/bitstream/handle/10183/49730/000658895.pdf?sequence=1

Lima, N. F. F., Santos, P. R. N., Pedrosa, M. S. \& Delboni, M. G. (2017) Cimentos biocerâmicos em endodontia: revisão de literatura. Revista da Faculdade de Odontologia UPF, 22(2), 248-254. https://doi.org/10.5335/rfo.v22i2.7398

Martins, C. R., Lopes, W. A. \& Andrade, J. B. (2013). Solubilidade Das Substâncias Orgânicas. Química Nova, 36(8), 1248-1255. 10.1590/S010040422013000800026

Mendes, A. T., Silva, P. B., Só, B. B., Hashizume, L. N., Vivan, R. R., Rosa, R. A., Duarte, M. A. H. \& Só, M. V. R. (2018). Evaluation of Physicochemical Properties of New Calcium Silicate - Based Sealer. Brazilian Dental Journal, 29(6), 536-540. doi: 10.1590/0103-6440201802088

Nascimento Sobrinho, G. A. (2014). Estudo da sorção e da contribuição da troca iônica na dinâmica do Cs em solos altamente intemperizados. [Dissertação, Instituto de Radioterapia de Dosimetria do Rio de Janeiro]. http://moodle.ird.gov.br/ensino/images/DissertacoesMestrado/Dissert .Mestrado2014/dissertao_guilherme\%20augusto\%20nascimento\%20sombrinho.pdf

Oliveira, P. H. C. (2017). Biocompatibilidade e capacidade de mineralização de um cimento Biocerâmico experimental. Estudo em tecido subcutâneo de ratos. [Trabalho de conclusão de curso, Faculdade de Odontologia de Araçatuba]. https://repositorio.unesp.br/handle/11449/156747

Razdan, A., Benetti, A. R. \& BJØRNDAL, L. (2019) Do in vitro solubility studies on endodontic sealers demonstrate a high level of evidence? A systematic review. Acta Odontologica Scandinavica, Scandinavia, 77(4), 253-263. 10.1080/00016357.2018.1538535

Santos, A. F. (2019). Avaliação da viabilidade celular de fibroblastos L929 e da resposta inflamatória em tecido subcutâneo de ratos ao novo material Bio-C Pulpo. [Dissertação, Universidade Federal de Alfenas]. https://bdtd.unifal-mg.edu.br:8443/handle/tede/1362

Sousa, N. B., Nunes, M. A. C., Veloso, K. M. M. \& Pereira, A. F. V. (2014). Agregado de trióxido mineral e uso como material retro-obturador em cirurgia paraendodôntica. Revista Brasileira de Odontologia, 71(2), 144-147. http://revodonto.bvsalud.org/scielo.php?script=sci_arttext\&pid=S003472722014000200006

Torres, F. F. E. (2016). Testes convencionais e empregando micro-ct na avaliação de propriedades físico-químicas de materiais retrobturadores e cimentos endodônticos. [Dissertação, Faculdade de Odontologia de Araraquara]. https://repositorio.unesp.br/handle/11449/138247

Viapiana, R., Flumignan, D. L., Guerreiro-Tanomaru, J. M., Camilleri, J. \& Tanomaru-Filho, M. (2013). Physicochemical and mechanical properties of zirconium oxide and niobium oxide modified Portland cement-based experimental endodontic sealers. International Endodontic Journal, 47(5), 437-448.: 10.1111/iej.12167

Vitti, R. P., Prati, C., Sinhoreti, M. A. C., Zanchi, C. H., Silva, M. G. S., Ogliari, F. A. \& Gandolfi, M. G. (2013). Chemical-physical properties of experimental root canal sealers based on butyl ethylene glycol disalicylate and MTA. Dental Materials, 29(12), 1287-1294. 10.1016/j.dental.2013.10.002

Zhou, H. M., Shen, Y., Zheng, W., Li, L., Zheng, Y. F. \& Haapasalo, M. (2013). Physical Properties of 5 Root Canal Sealers. Journal of Endodontics, 39(10), 1281-1286. 10.1016/j.joen.2013.06.012

Zordan-Bronzel, C. L., Torres, F. F. E., Tanomaru-Filho, M., Chávez-Andrade, G. M., Bosso-Martelo, R. \& Guerreiro-Tanomaru, J. M. (2019). Evaluation of Physicochemical Properties of a New Calcium Silicate-based Sealer, Bio-C Sealer. Journal of Endodontics, 45(10), 1248-1252. 10.1016/j.joen.2019.07.006

Zvi, M., Bettina, B. \& Harold, E. G. (2011) Instrumentos, Materiais e Aparelhos. In: K. M. Hargreaves \& S. Cohen (10a ed.), Caminhos da Polpa (pp. 250264). Elsevier.) 